\title{
Influence of maternal undernutrition and stage of gestation on perirenal adipose tissue development and gene expression in fetal sheep: a putative presage of later adiposity
}

\author{
J. M. Wallace, J. S. Milne, R. P. Aitken and C. L. Adam \\ Rowett Institute of Nutrition and Health, University of Aberdeen, Aberdeen, AB21 9SB, UK
}

In precocial mammals appropriate prenatal adipose tissue development is essential for adequate thermoregulation at birth to ensure immediate survival. In addition, adipose tissue is central to energy metabolism throughout the life-course and derangements in its early development potentially impact body composition in later life. Accordingly, adipose tissue is widely considered to be a key target of developmental programming by maternal and /or fetal undernutrition ${ }^{(1)}$. Herein we investigated the impact of maternal undernutrition on fetal adiposity and expression of genes regulating fetal perirenal adipose tissue (PAT) development and function at gestation days 89 and $130($ term $=145 \mathrm{~d})$.

Singleton fetuses derived by single sire embryo transfer were taken from adolescent dams fed a control (C) intake to maintain adiposity throughout pregnancy or undernourished (UN) to maintain conception weight but deplete maternal reserves ( $n$ 7/group/stage). Fetal PAT was harvested and mRNA expression of genes $(n 13)$ involved in adipocyte proliferation, differentiation and function were measured by quantitative real time RT-PCR. At day 130 the density of unilocular and multilocular adipocytes in PAT was quantified in $H \& E$ stained wax sections and the fat content of the fetal carcass was determined by chemical analysis.

By design the external adiposity score of $\mathrm{C}$ dams was maintained throughout. In contrast the change in adiposity score for UN dams was equivalent to average losses of $6 \cdot 2$ and $8.8 \%$ body fat at days 89 and 130 , respectively $(\mathrm{P}<0.001)$. Fetal weight was independent of maternal nutrition at day 89 but by day 130 fetuses from UN dams were $17 \%$ lighter $(\mathrm{P}<0.005)$. By late gestation UN fetuses had reduced carcass fat percentage and lower absolute and fetal weight-specific PAT mass containing fewer unilocular adipocytes $(\mathrm{P}<0 \cdot 05)$. Relative PAT expression of $I G F 1, I G F 2, I G F 2 R$ and peroxisome-proliferator-activated receptor-gamma $(P P A R G)$ mRNA was lower in UN than in $\mathrm{C}$, predominantly at day $89(\mathrm{P}<0.05)$. Independent of maternal nutrition, PAT gene expression of PPARG, glycerol-3-phosphate dehydrogenase, hormone sensitive lipase, leptin, uncoupling protein-1 and prolactin receptor increased and four IGF system genes decreased between 89 and 130 days $(\mathrm{P}=0.003-<0.001)$. Fatty acid synthase and lipoprotein lipase $(L P L)$ mRNAs were not influenced by nutrition or stage of pregnancy. Females had higher $L P L$ and leptin mRNA than males $(\mathrm{P}=0.002)$ and $L P L$, leptin and $P P A R G$ mRNAs were decreased by $\mathrm{UN}$ at day 89 in females only $(\mathrm{P}<0.05)$. PAT gene expression correlations with PAT mass were stronger at day 89 than day 130.

These data support the hypothesis that molecular markers of fetal adipose tissue development are temporally sensitive to maternal undernutrition in precocial mammals. Some key genes regulating adipose tissue development and function are active from midgestation when they are variously sensitive to maternal undernutrition leading to reduced fetal adiposity in late gestation. Furthermore, gender differences in adipose tissue gene expression emerge in fetal life. Whether these prenatal events impact body composition in adult life remains unknown but it is clear that adequate maternal nutrition from the earliest stages of pregnancy is essential to optimise fetal growth and neonatal adipose tissue development.

1. Lukaszewski et al. (2013) AJP Endo Metab 305, E1195-1207. 